\title{
Flavonoids play a key role in resistance to accumulation of aflatoxin in corn
}

Lina Castano-Duque, Brian M Mack, Matthew K Gilbert, Christine M Sickler, Jeffrey W Cary, Kanniah Rajasekaran Lina.Castano.Duque@usda.gov of @LinaCastano_Dy

\section{Research goal}

Can we find a way to identify multiple key elements and pathways of corn resistance to Aspergillus flavus by doing major data integration analysis?

\section{Importance}

Aspergillus flavus is a fungus with many traits. This fungus colonizes crops such as corn, cottonseed, peanut and more. Some strains produce highly toxic and carcinogenic chemicals such as aflatoxin. Corn resistance to aflatoxin accumulation is a polygenic trait.

\section{Objectives and experiments}

Obj. 1. Use GWAS phenotypic data to identify genes and pathways associated with corn resistance to aflatoxin accumulation

Obj. 2. Perform post-GWAS using RNA-seq data from kernel screening assay (KSA) of TZAR102 (Resistant line) and Va35 (Susceptible line)

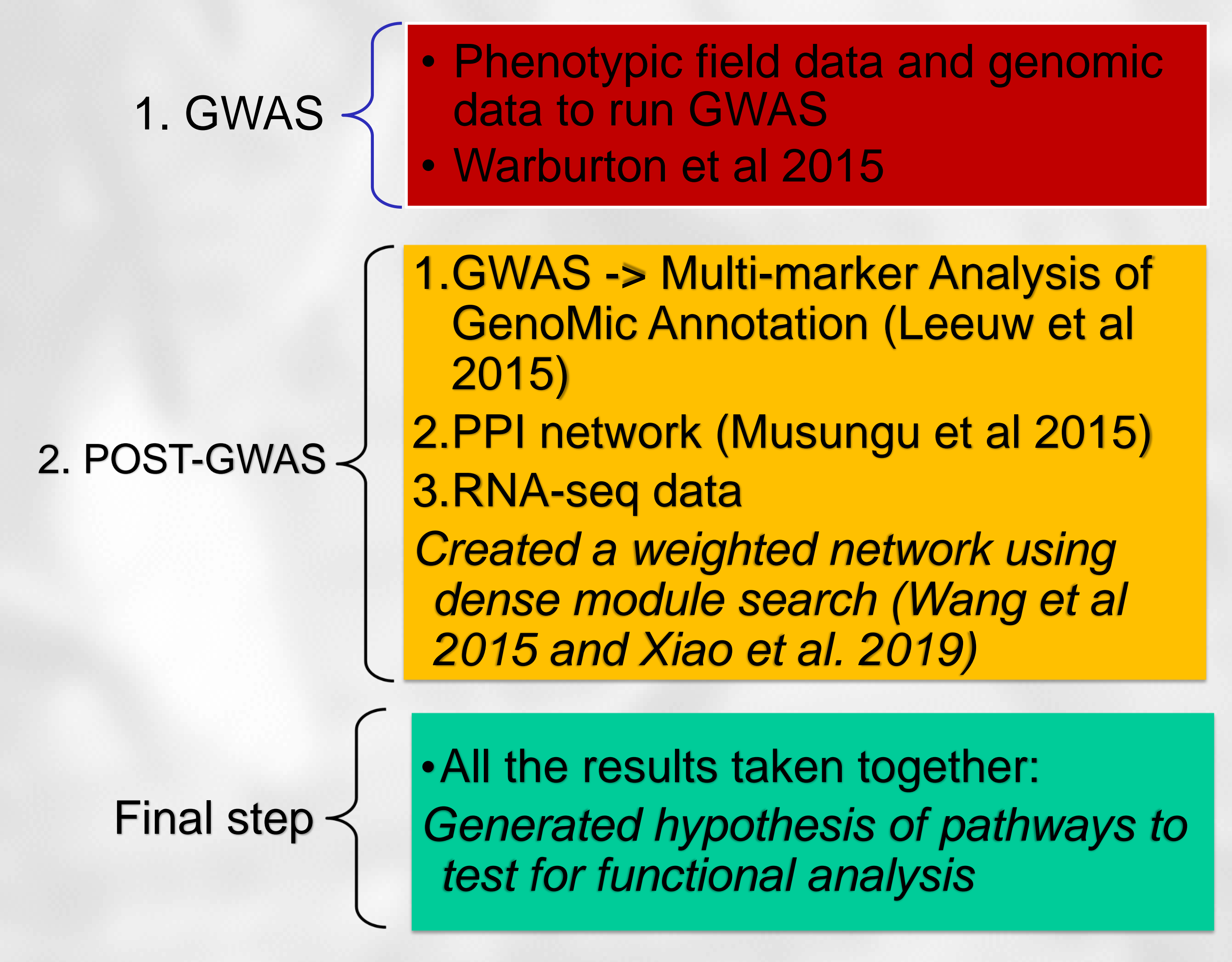

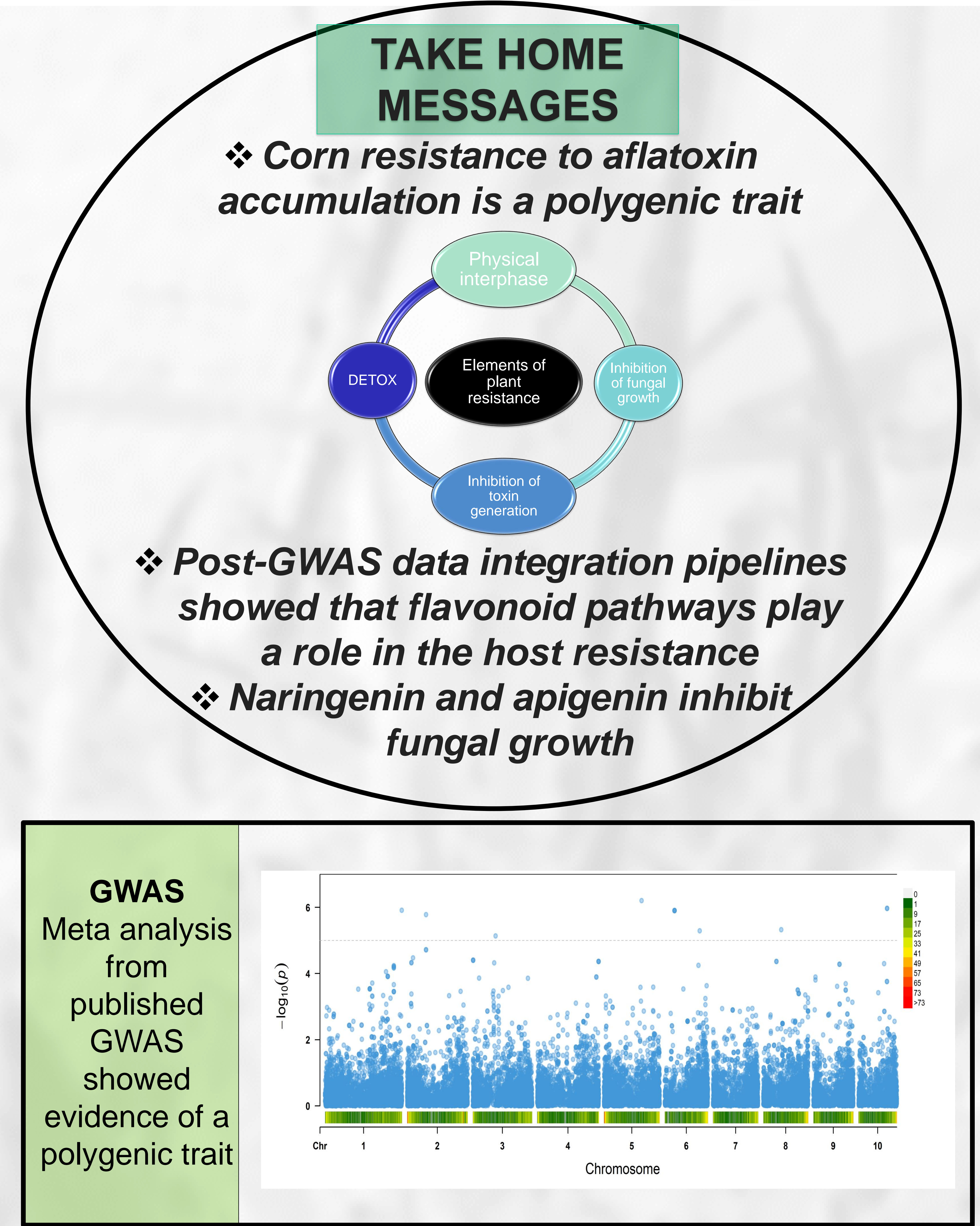
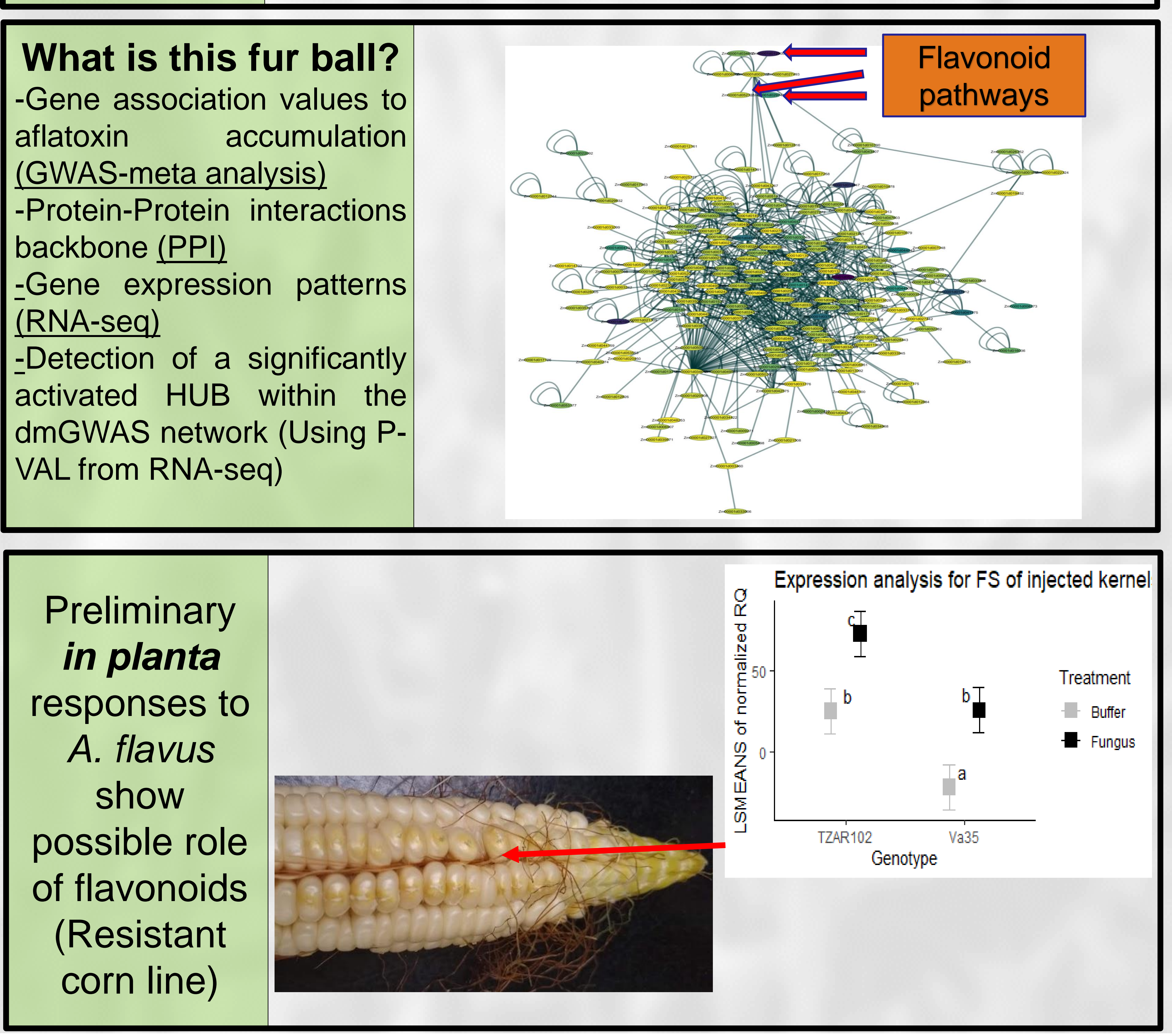

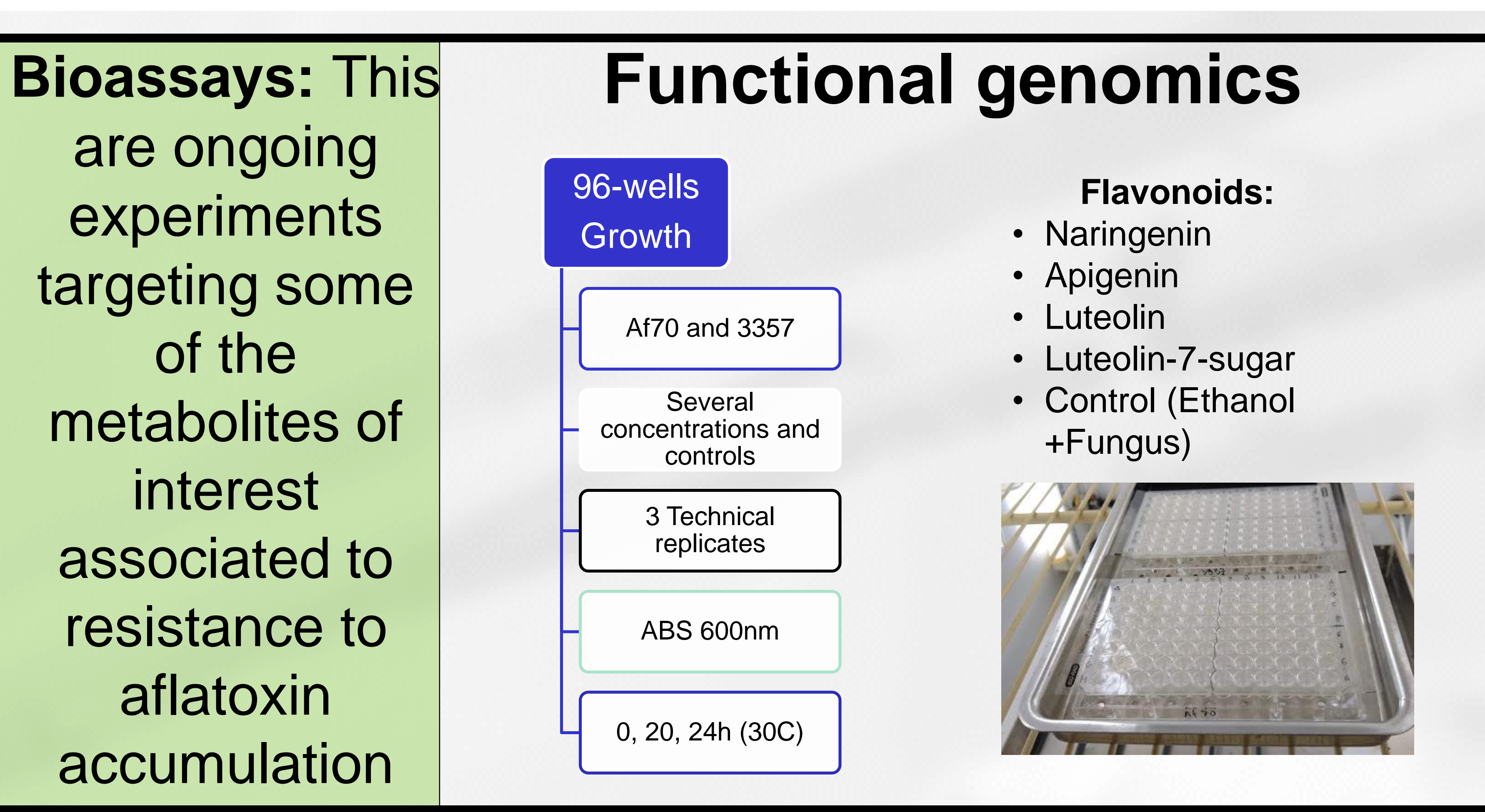

accumulation

Preliminary results showed:

Naringenin and apigenin have a high relationship with inhibition of fungal growth when incubated with $A$. flavus 70 and 3357 strains

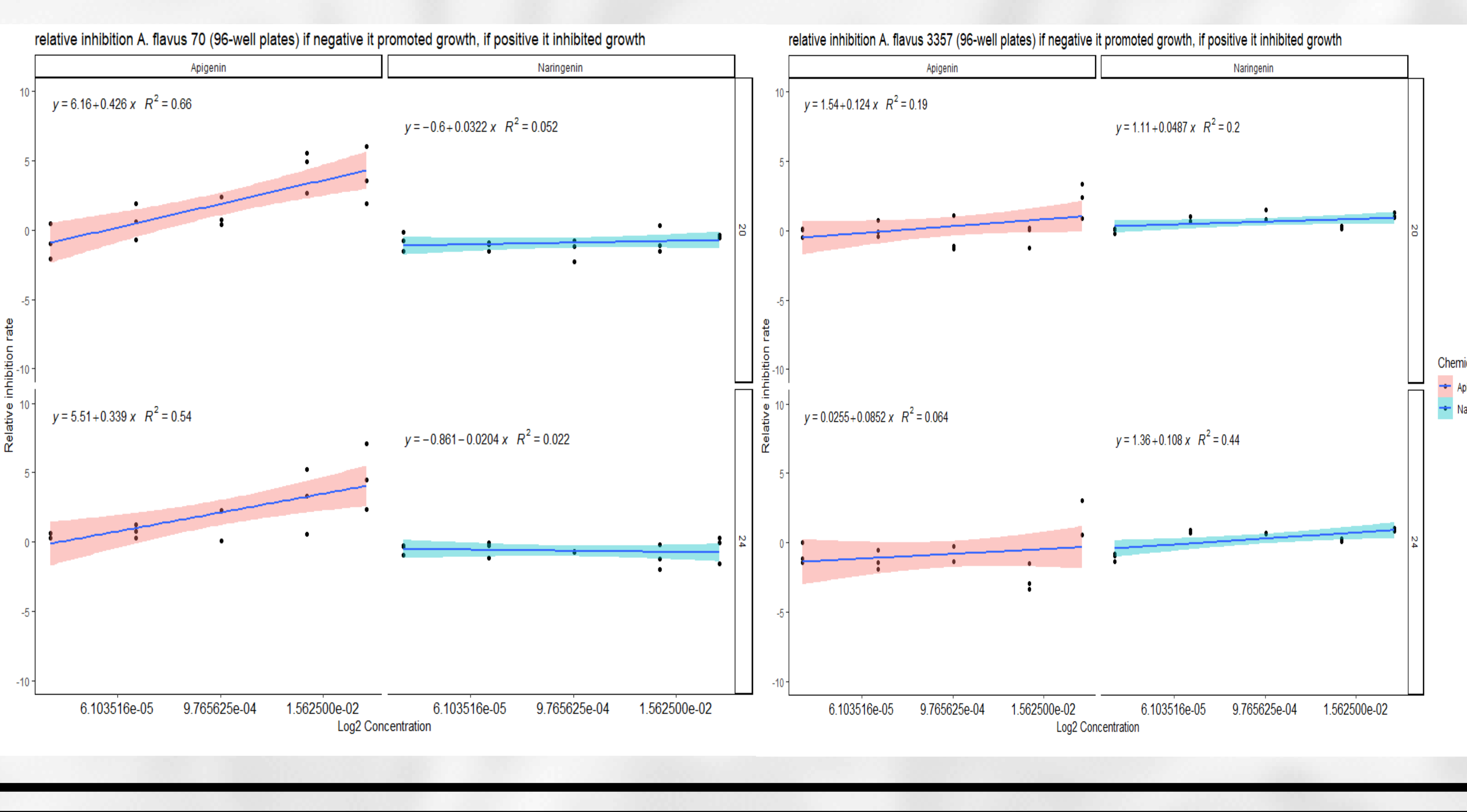

We do not know:

$\square$ The directionality of the response

DThe metabolite profile of the plant when infected (Flavones, flavonol and others)

We know that flavonoid pathways are involved in the corn response to $A$. flavus infection

$\square$ The effect of flavonoids over the fungus

\section{What to do?}

* Flavonoid profiles in planta assays -> Ongoing

* Fungal bioassays -> Ongoing

\section{Acknowledgments}

All the member of USDA-ARS SRRC Food and feed safety unit (Gilbert, Rajasekaran, Lebar, Cary, Sweany, Downey and many more)

All the member of SRRC in New Orleans

Paul Williams USDA-ARS research unit at Mississippi State 